\section{IDENTIFYING PRIORITIES FOR IMPROVED CHILD HEALTHCARE: A MIXED METHODS ANALYSIS OF SAFETY INCIDENT REPORTS}

Philippa Rees, ${ }^{1}$ Adrian Edwards, ${ }^{1}$ Colin Powell, ${ }^{2}$ Huw Williams, ${ }^{1}$ Peter Hibbert, ${ }^{3}$ Meredith Makeham, ${ }^{3}$ Donna Luff, ${ }^{4}$ Gareth Parry, ${ }^{5}$ Aziz Sheikh, ${ }^{6}$ Liam Donaldson, ${ }^{7}$ Andrew Carson-Stevens ${ }^{1} .{ }^{1}$ Cochrane Institute of Primary Care and Public Health, Cardiff University, United Kingdom-Wales; ${ }^{2}$ Department of Child Health, Institute of Molecular \& Experimental Medicine Cardiff University, United Kingdom-Wales; ${ }^{3}$ Australian Institute for Healthcare Innovation, Macquarie University, Australia; ${ }^{4}$ Institute for Professionalism and Ethical Practice/Department of Anesthesia Boston Children's Hospital, United States; ${ }^{5}$ Institute for Healthcare Improvement, United States; ${ }^{6}$ Centre for Population Health Sciences, University of Edinburgh, United Kingdom-Scotland; ${ }^{7}$ Imperial College London, United Kingdom-England

\subsection{6/bmjqs-2015-IHlabstracts. 16}

Background Globally children are failed on numerous indicators of care quality, most of which is provided in the primary care setting. There is a paucity of research and development to explain iatrogenic harm in the primary care setting, not least safety events involving children.

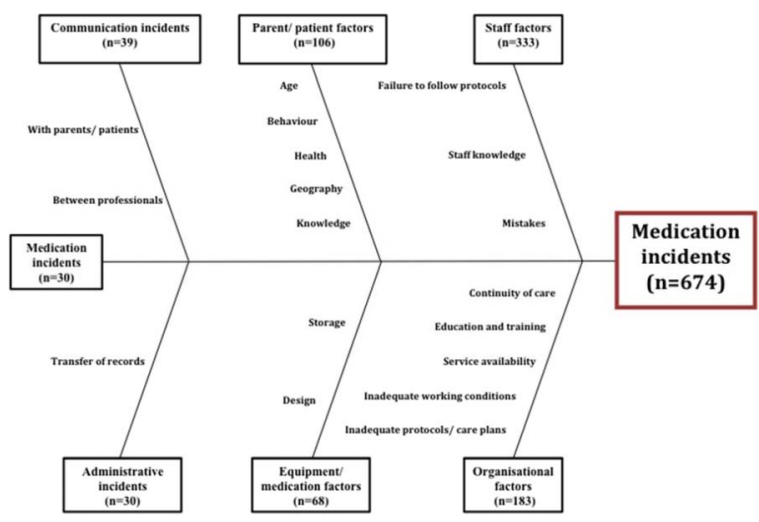

Figure 1 Ishikawa diagram illustrating the factors and incidents frequently reported as contributing to medication incidents.

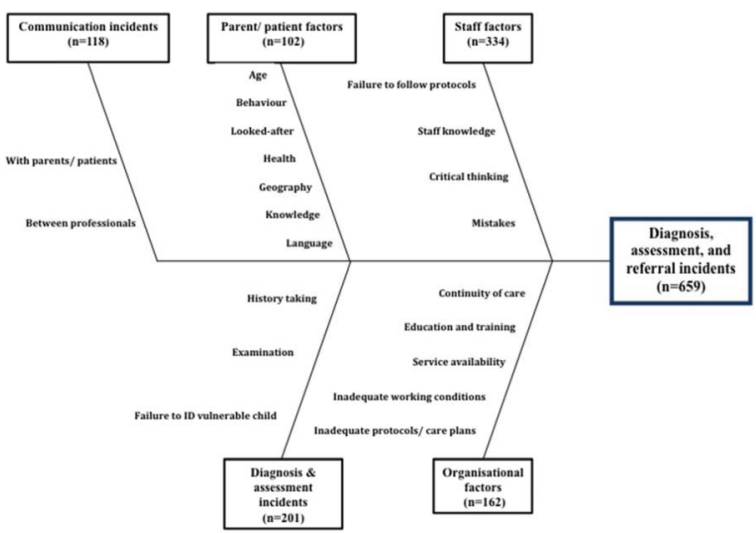

Figure 2 Ishikawa diagram illustrating the factors and incidents frequently reported as contributed to diagnosis, assessment, and referral incidents.

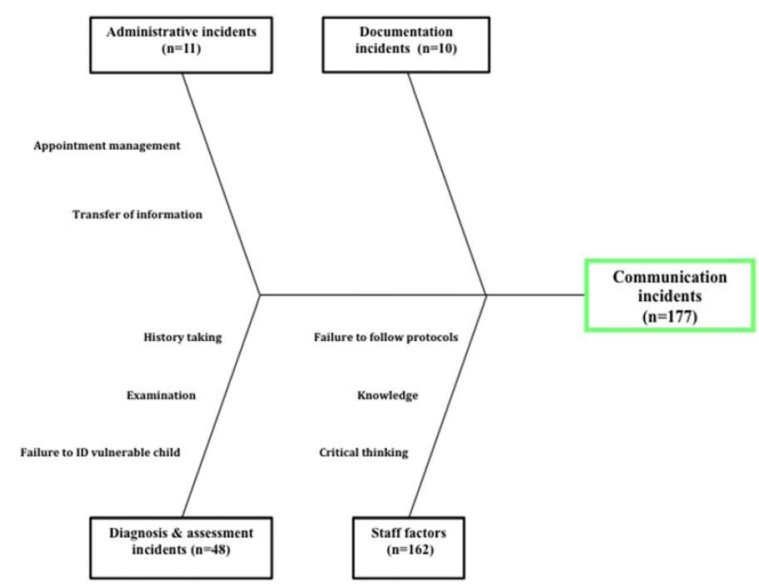

Figure 3 Ishikawa diagram illustrating the factors and incidents frequently reported as contributing to communication incidents.

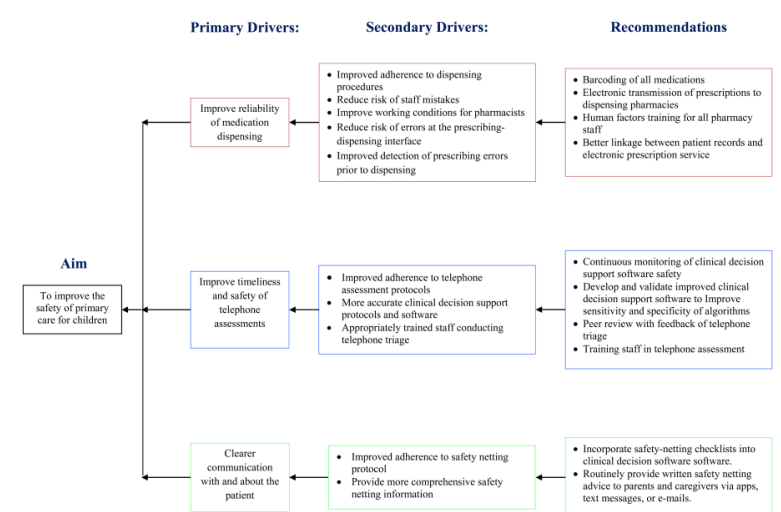

Figure 4 Driver diagram illustrating opportunities to improve the safety of primary care for children.

Objectives We identified priority issues (by frequency and level of harm) reported by clinicians from the England and Wales National Reporting and Learning System (NRLS). The concepts and content within reports empirically informed a systems-level Driver diagram for improvement of child health. 
Methods We undertook a retrospective cross-sectional mixed methods study of safety incident reports from primary care involving 'unwell' children between 2003-2013. We classified incident types, contributory factors, and harm outcomes. Data underwent exploratory analysis to explore the relationship between variables, and thematic analysis provided in-depth contextual insights. Subject matter experts identified key drivers for improvement.

Results Of 2191 incidents identified, priority improvement areas included: medication provision in the community pharmacy setting; diagnosis, assessment, and timely referral of acutely unwell children in out of hours care settings; and communication with and about the child. Reported causes are outlined in the attached Ishikawa diagrams (figures 1-3) and the related Driver diagram (figure 4) summarises opportunities to mitigate priority concepts.

Conclusions Analysis of incident reports can be used to inform national-level improvement initiatives when there is a paucity of existing available evidence. Further validation and development of the proposed designs are needed with the clinical teams responsible for care delivery. 